\title{
Optical phase conjugation for turbidity suppression in biological samples
}

\author{
ZAHID YAQOOB ${ }^{1 \dagger}$, DEMETRI PSALTIS 1,2, MICHAEL S. FELD³ AND CHANGHUEI YANG'* \\ ${ }^{1}$ Electrical Engineering, California Institute of Technology, 1200 East California Boulevard, Pasadena, California 91125, USA \\ ${ }^{2}$ School of Engineering, Ecole Polytechnique Fédérale de Lausanne, CH-1015 Lausanne, Switzerland \\ ${ }^{3}$ Massachusetts Institute of Technology, G. R. Harrison Spectroscopy Laboratory, 77 Massachusetts Avenue 6-207, Cambridge, Massachusetts 02139, USA \\ tPresent address: Massachusetts Institute of Technology, G. R. Harrison Spectroscopy Laboratory, 77 Massachusetts Avenue 6-208, Cambridge, \\ Massachusetts 02139, USA \\ *e-mail: chyang@caltech.edu
}

Published online: 27 January 2008; doi:10.1038/nphoton.2007.297

Elastic optical scattering, the dominant light-interaction process in biological tissues, prevents tissues from being transparent. Although scattering may appear stochastic, it is in fact deterministic in nature. We show that, despite experimental imperfections, optical phase conjugation $(\lambda=532 \mathrm{~nm})$ can force a transmitted light field to retrace its trajectory through a biological target and recover the original light field. For a $0.69-\mathrm{mm}$-thick chicken breast tissue section, we can enhance pointsource light return by a factor of $\sim 5 \times 10^{3}$ and achieve a light transmission enhancement factor of 3.8 within a collection angle of $29^{\circ}$. Additionally, we find that the reconstruction's quality, measured by the width of the reconstructed point source, is independent of tissue thickness (up to a thickness of $0.69 \mathrm{~mm}$ ). This phenomenon may be used to enhance light transmission through tissue, enable measurement of small tissue movements, and form the basis of new tissue imaging techniques.

Elastic optical scattering in biological tissue typically dominates over absorption by an order of magnitude or more. As a point of reference, consider light propagation in chicken breast tissue at a wavelength of $633 \mathrm{~nm}$ - the scattering coefficient is $23 \mathrm{~mm}^{-1}$ and the reduced scattering coefficient $0.8 \mathrm{~mm}^{-1}$, but the mean absorption coefficient is only $0.01 \mathrm{~mm}^{-1}$ (ref. 1). Being the dominant light-interaction process, scattering prevents tissue from being transparent by distorting and effectively randomizing the transmission light-field paths. Yet, it is also known that elastic optical scattering is a deterministic and time-reversible process. In other words, if we can record the phase and amplitude of the propagating scattered light field completely and reproduce a backpropagating phase-conjugated (or time-reversed) field, this field should be able to retrace its trajectory through the scattering medium and return the original input light field. More than 40 years ago it was demonstrated that optical phase conjugation (OPC) could reverse optical scattering induced by a ground glass slide ${ }^{2}$. Although OPC has been used in numerous laser-related applications, such as high-resolution imaging ${ }^{3}$, laser resonators ${ }^{4,5}$ and pulse compression ${ }^{6}$, its use in suppressing turbidity in biomedical applications has remained largely unexplored. The use of interferometric wavefront sensing and holographic techniques in biomedical optics has largely focused on phase imaging and the selection of minimally scattered light for imaging ${ }^{7-9}$. We note that there have been significant investigations of phase-conjugation techniques in the microwave and ultrasound regimes. Their uses range from enhancing acoustic energy delivery to target brain tumours or kidney stones ${ }^{10}$ to enabling subwavelength focusing of microwaves to increase the information transfer rate per unit volume ${ }^{11}$.

Most of these techniques have the potential to be translated into optical regime equivalents for biomedical applications if OPC can suppress scattering in biological tissues. To accomplish this, three challenges must be addressed. First, as tissues are highly scattering, light propagating beyond a certain tissue thickness $(>100 \mu \mathrm{m}$ in general) is predominantly multiply scattered. In our experiments, the mean number of scattering events per photon is as high as 26 for a $0.69-\mathrm{mm}$-thick chicken breast tissue section. Previous optical experiments did not come close to this level of turbidity. It is an open question as to whether turbidity suppression in tissue through OPC is observable, as the effect can be expected to be more prone to noise and sensitive to the reconstruction condition as scattering increases. Second, it is not feasible to record the entire scattered light field. There is a recording angle range associated with most forms of optical wavefront recording media, such as holographic plates, photorefractive crystals and so on, beyond which the recording efficiency declines significantly. In addition, a fraction of the light field illuminating a sufficiently thick tissue section will be backscattered. As such, it is reasonable to question whether an incompletely phase-conjugated light field can adequately retrace its trajectory in the biological target. Third, some of the light will be absorbed by the tissue. This breaks the time direction symmetry associated with that light component.

This paper presents a first-of-its-kind study of OPC for turbidity suppression in biological tissues, which we call 'turbidity suppression by optical phase conjugation' (TSOPC). We show that, although the above three factors can affect the quality and extent of the reconstruction, turbidity suppression can nevertheless be observed for sections of chicken breast tissue up to a thickness of $0.69 \mathrm{~mm}$ at $\lambda=532 \mathrm{~nm}$. We also characterize the key features associated with this phenomenon. 


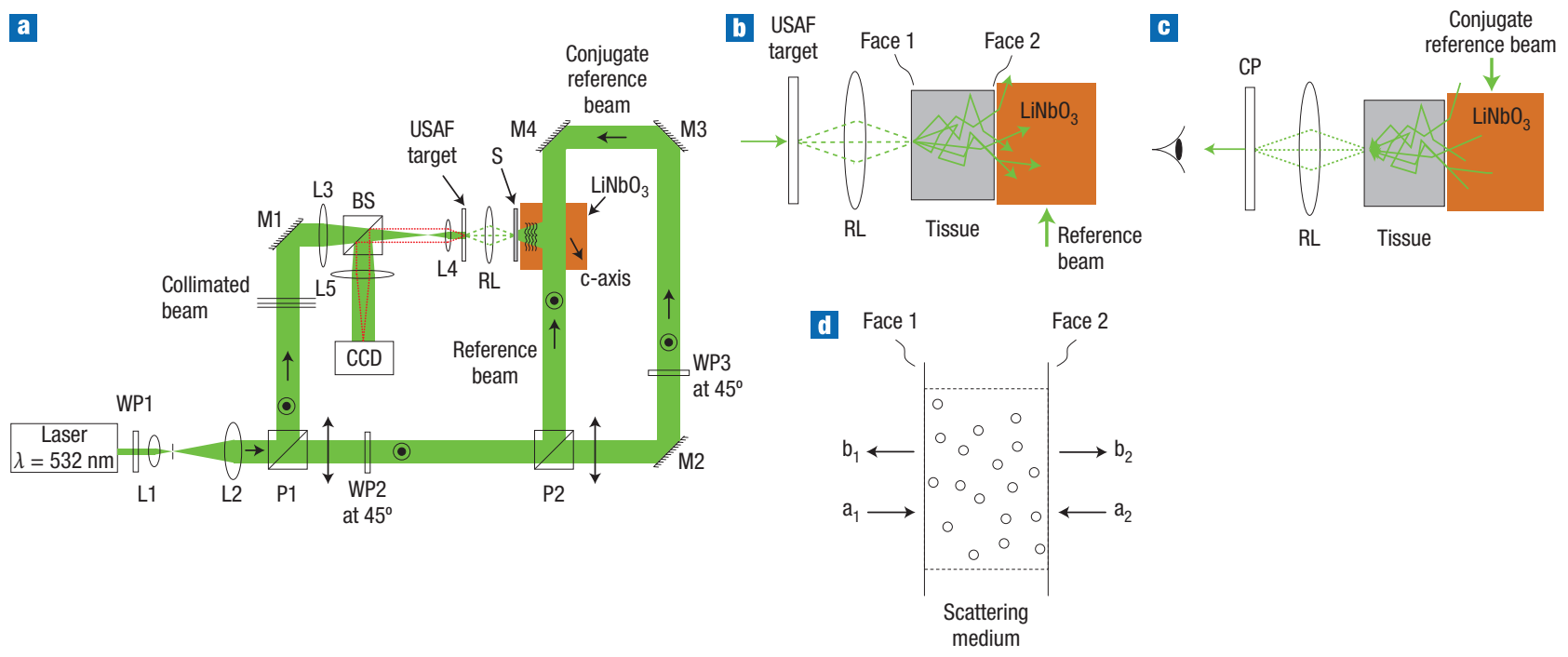

Figure 1 Schematics of TSOPC set-up and scattering medium. a, Experimental set-up to confirm the TSOPC phenomenon in biological tissues. The concentric (black) dots and circles represent vertical polarization, and the double-ended arrow in the plane of the paper symbolizes horizontal polarization. b,c, Schematics for recording of tissue turbidity information (b) and reconstruction of the OPC light field (c), respectively. $\mathbf{d}$, Schematic of a scattering medium. $\mathrm{a}_{j}$ and $\mathbf{b}_{i}$ are the complex incident and scattered fields, respectively, at the ith face of the scattering medium. $L i$, ith spherical lens; RL, relay lens; CP, compensation plate; Mi, ith mirror; WPi, ith half-wave plate; $\mathrm{P} i$, ith polarization beam splitter; BS, 50/50 beam splitter; S, scattering sample.

\section{RESULTS}

\section{TURBIDITY SUPPRESSION BY OPTICAL PHASE CONJUGATION}

The salient features of TSOPC are well illustrated in the first experiment. We used a photorefractive $45^{\circ}$-cut $0.075 \%$ Fe-doped $\mathrm{LiNbO}_{3}$ crystal as the OPC light-field generator or phase-conjugate mirror (PCM). The recording and playback scheme (Fig. 1a) is detailed in the Methods. In this study, our target was a $0.46-\mathrm{mm}$ thick chicken breast tissue section. Light at $\lambda=532 \mathrm{~nm}, 3.5 \mathrm{~mW}$ power and with a $1 / \mathrm{e}^{2}$ beam size of $0.7 \mathrm{~mm}$ was transmitted through a standard negative United States Air Force (USAF) target, that is, a resolution test slide with a clear pattern on a chrome background. The patterned light was then imaged onto the front face (face 1, Fig. 1b) of the tissue section (S) using a 1:1 imaging relay lens. The forward-scattered light traversed the tissue sample, exited from face 2, and arrived at the photorefractive crystal for holographic recording. The recording geometry is examined more closely in Fig. 1b. In the experiment, the separation between the crystal facet and the tissue section was $0.5 \mathrm{~mm}$. The $7.8-\mathrm{mm} 1 / \mathrm{e}^{2}$ diameter, $6.5-\mathrm{mW}$ reference beam used during the recording process crossed the crystal at a $1-\mathrm{mm}$ distance from the crystal facet facing the tissue section. This implies a nominal maximum recording angle range of $66^{\circ}$. The hologram recording time was $\sim 2$ min for this experiment. On completion, the USAF target was replaced with a compensation glass plate, and an OPC light field was generated with a conjugate reference beam of $12 \mathrm{~mW}$. This light field retraced the path of the original transmission and recreated an image of the USAF target on face 1 of the tissue (Fig. 1c). This image was then relayed onto a CCD camera (DMK 31BF03, The Imaging Source).

Mathematically, the interaction of the incident light field $\left(\begin{array}{c}\mathbf{a}_{1} \\ \mathbf{a}_{2}\end{array}\right)$ with a scattering medium can be expressed as ${ }^{12}$ (Fig. 1d)

$$
\left(\begin{array}{l}
\mathbf{b}_{1} \\
\mathbf{b}_{2}
\end{array}\right)=\left(\begin{array}{ll}
\bar{S}_{11} & \bar{S}_{12} \\
\bar{S}_{21} & \bar{S}_{22}
\end{array}\right)\left(\begin{array}{l}
\mathbf{a}_{1} \\
\mathbf{a}_{2}
\end{array}\right)
$$

where $\left(\begin{array}{ll}\bar{S}_{11} & \bar{S}_{12} \\ \bar{S}_{21} & \bar{S}_{22}\end{array}\right)$ is the scattering matrix associated with the medium, $\left(\begin{array}{l}\mathbf{b}_{1} \\ \mathbf{b}_{2}\end{array}\right)$ is the output light field and $\mathbf{a}_{2}=0$. The subscript denotes the terminal face 1 or 2 of the scattering medium. In this case, the light field impinging on the PCM is given by $\bar{S}_{21} \mathbf{a}_{1}$. The OPC light field travelling back towards face 2 of the tissue section can be expressed as

$$
\psi_{\text {phase conjugate }}=\bar{A} \bar{S}_{21}^{*} \mathbf{a}_{1}^{*}
$$

where $\bar{A}$ represents the reduction in angular range of the reconstructed wave owing to the incomplete recording and playback of the transmitted wave. The reconstructed light field on face 1 of the tissue section can be written as

$$
\psi_{\text {reconstructed }}=\bar{S}_{12} \bar{A}_{S_{21}^{*}} \mathbf{a}_{1}^{*} .
$$

As the relationship between any two points on the medium's surface is symmetrical we obtain $\bar{S}_{21}=\bar{S}_{12}^{T}$.

In an ideal case, the capture of the initial light transmission is complete, which leads to a reduction of $\bar{A}$ to a unitary matrix. Also, the medium in such a case is lossless and backscattering is absent-this leads to $\bar{S}_{12} \bar{S}_{12}^{\dagger}=I$ by energy conservation, where $\bar{S}_{12}^{\dagger}$ is the complex conjugate of $\bar{S}_{12}^{T}$. The reconstructed light-field expression can then be written as

$$
\psi_{\text {ideal, reconstructed }}=\bar{S}_{12} \bar{S}_{12}^{\dagger} \mathbf{a}_{1}^{*}=\mathbf{a}_{1}^{*} .
$$

The extent to which an experimental realization approaches this ideal is verified in our experiment (Fig. 2). Figure 2a shows USAF target imaging through a $0.46-\mathrm{mm}$-thick agarose section and Fig. $2 \mathrm{~b}$ for a tissue section of the same thickness, using plane-wave illumination. At $\lambda=532 \mathrm{~nm}$, the chicken breast tissue scattering coefficient was $38 \mathrm{~mm}^{-1}$ (quantified through interferometric measurement of ballistically propagating transmission through tissue) and the sum of the reduced scattering coefficient and the 

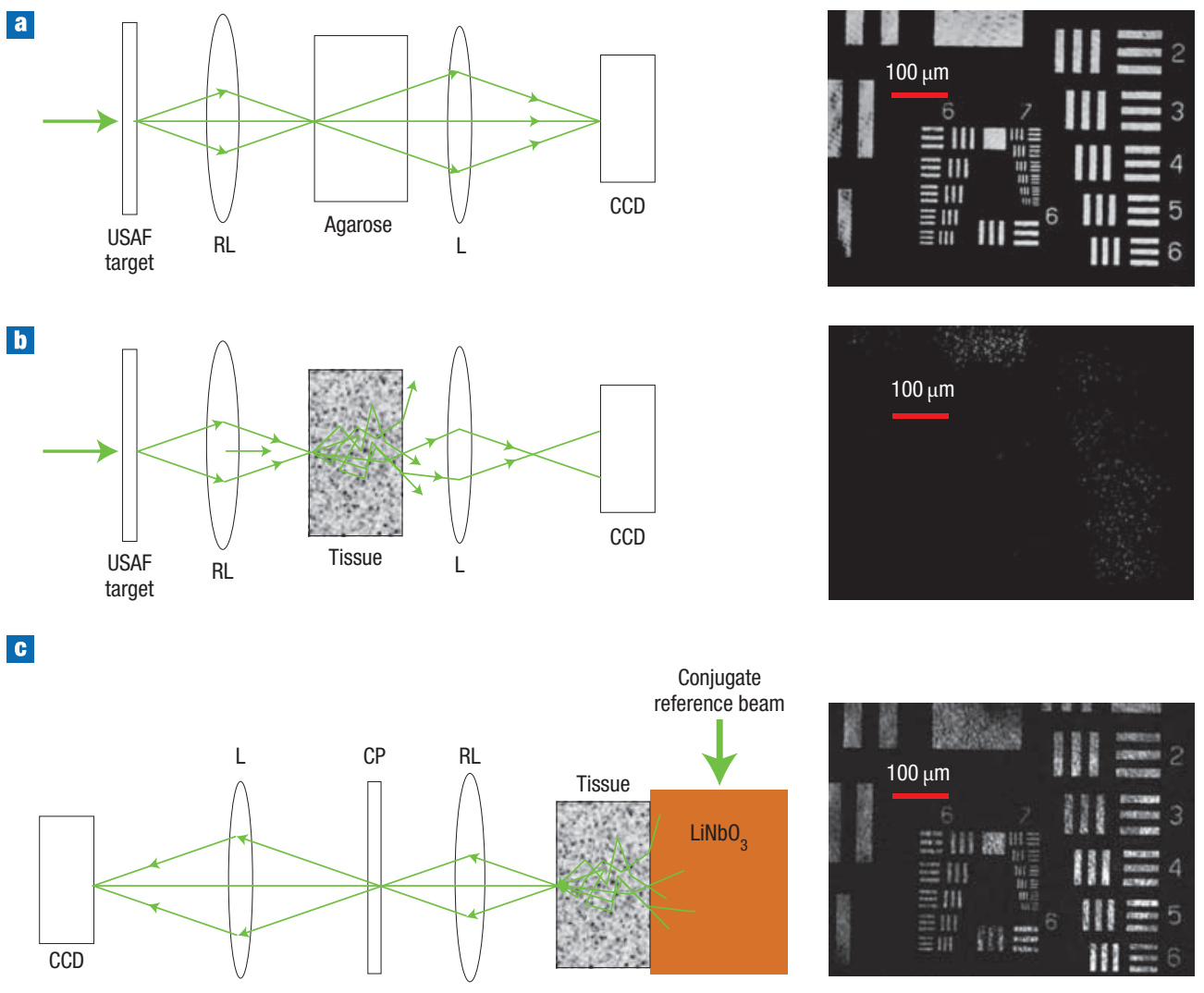

Figure 2 Demonstration of the TSOPC phenomenon through a 0.46-mm-thick chicken breast tissue section. a,b, Imaging of USAF target through 0.46-mm-thick agarose (a) and chicken breast tissue (b) sections, using plane-wave illumination. c, Reconstruction of USAF target image through 0.46-mm-thick chicken breast tissue using the OPC light field. The high quality of the reconstructed image attests to the fact that the OPC light field can indeed suppress turbidity by retracing its initial trajectory through the tissue. RL, relay lens; L, imaging lens; $C P$, compensation plate. Note that in all cases the images are brought to the sharpest possible focus.

absorption coefficient was $0.45 \mathrm{~mm}^{-1}$ (quantified through transmission measurement). This implies that, on average, a photon is scattered 17 times in a $0.46-\mathrm{mm}$-thick tissue sample, and $19 \%$ of the input light did not reach face 2 of the $0.46-\mathrm{mm}$ thick tissue section. The high quality of the reconstructed image through the $0.46-\mathrm{mm}$ thick tissue section in Fig. $2 \mathrm{c}$ demonstrates that the conjugated signal beam can indeed retrace its initial trajectory through the tissue to a good degree, in spite of these issues.

\section{POINT-SOURCE ILLUMINATION}

The next set of experiments studied the TSOPC phenomenon in a more quantitative fashion. In this case, we focused a $0.65-\mathrm{mW}$ signal beam to a $1.4-\mu \mathrm{m} 1 / \mathrm{e}^{2}$ diameter spot using an L3 $($ Olympus PLAN $\times 10)$ at face 1 of the tissue section (Fig. 3a).

Each experiment consisted of the following. The transmitted light through the tissue section was first holographically recorded in the photorefractive crystal for $30 \mathrm{~s}$ with a reference beam power of $33 \mathrm{~mW}$. Next, a conjugate reference beam $(3.3 \mathrm{~mW})$ was used to generate the OPC light field, which travelled back through the tissue section and reconstructed the original light spot at face 1 of the tissue section. We displaced the tissue sample laterally in incremental steps to a limit of $8 \mu \mathrm{m}$ and acquired an image of the reconstructed spot for each displacement. Four targets were used-a 0.23-mm-thick clear agarose section (non-scattering control) and chicken breast tissue sections of thickness $0.23 \mathrm{~mm}, 0.46 \mathrm{~mm}$ and $0.69 \mathrm{~mm}$. The experiment was repeated eight times for each sample thickness. For the agarose section, the average return power through the sample was measured as $0.4 \mathrm{nW}$. This value was a function of the readout reference beam power and the crystal's recording efficiency. To eliminate contributions of these two experimental parameters from our analysis, we normalized our data with respect to our measurements from the agarose control.

We found that, under these experimental conditions, the strength of the recorded hologram decreased minimally over the experimental time frame of $\sim 2$ min for each TSOPC experiment (see Supplementary Information, Fig. S1) and, as such, does not have a significant impact on our findings. We also note that the efficiency of the TSOPC reconstruction is expected to drop as the biological samples change in time, but experimentally we did not observe a significant change over the time in which each experiment was conducted.

Figure $3 \mathrm{~b}-\mathrm{f}$ illustrates the average radial light intensity distribution in the reconstructed spot for $0 \mu \mathrm{m}, 2 \mu \mathrm{m}, 4 \mu \mathrm{m}, 6 \mu \mathrm{m}$ and $8 \mu \mathrm{m}$ displacements, respectively, of the 0.23 -mm-thick agarose sample (magenta) and the tissue sections of thickness $0.23 \mathrm{~mm}$ (black), $0.46 \mathrm{~mm}$ (red) and $0.69 \mathrm{~mm}$ (blue). Two observations are noteworthy. First, although a significant tissue section displacement leads to a mismatch between the scattering structures and the returning OPC light field, and results in no turbidity suppression, it can be observed from Fig. $3 \mathrm{c}$ that this phenomenon is tolerant to small sample displacements, particularly for thinner samples. Second, Fig. 3b illustrates that the width of the fully reconstructed spot appears surprisingly similar for all tissue thicknesses.

Figure $4 \mathrm{a}$ shows the normalized peak intensity as a function of sample lateral displacement. Several observations are worth noting. 

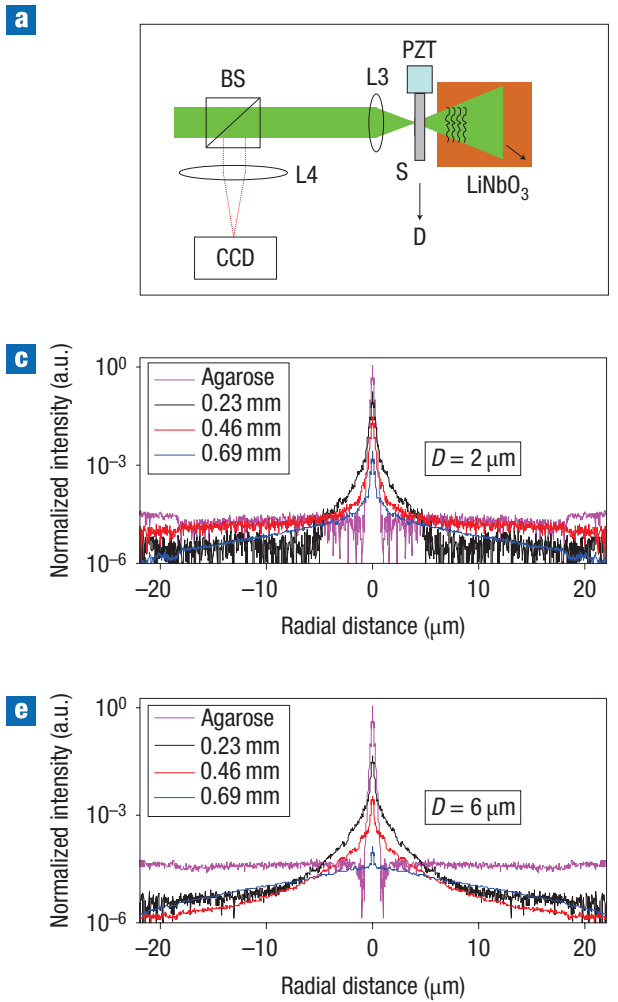
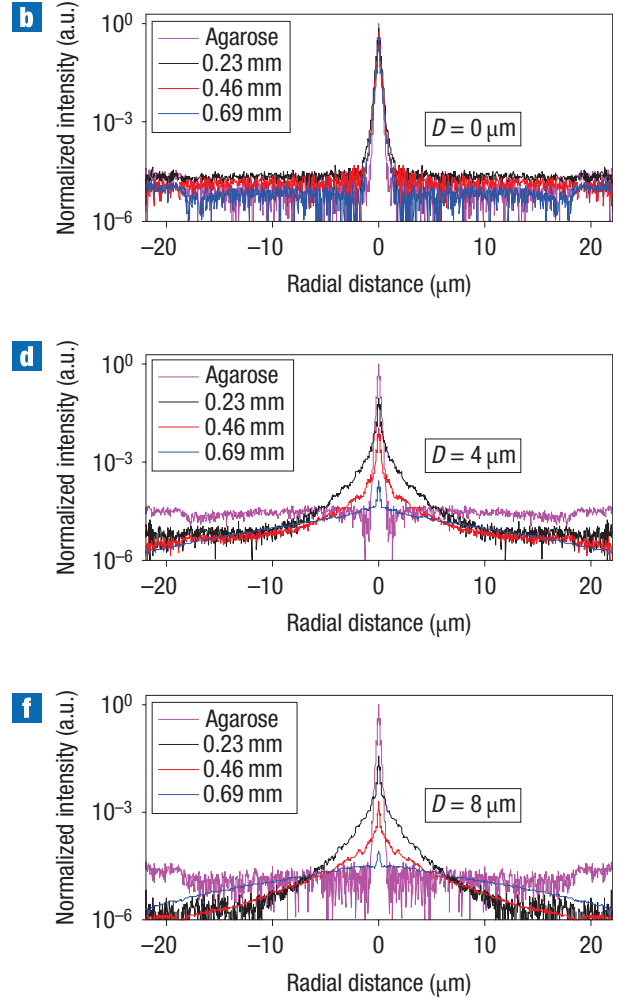

Figure 3 TSOPC using point-source illumination. a, Schematic of TSOPC experimental set-up. $\mathbf{b}-\mathbf{f}$, Average radial light intensity distributions of the reconstructed spots for $0.23-\mathrm{mm}$ agarose and $0.23-\mathrm{mm}$-, $0.46-\mathrm{mm}$ - and 0.69 -mm-thick chicken breast tissue sections at $0 \mu \mathrm{m}, 2 \mu \mathrm{m}, 4 \mu \mathrm{m}, 6 \mu \mathrm{m}$ and $8 \mu \mathrm{m}$ displacements, respectively. PZT, lead zirconate titanate actuator; S, sample; D, displacement of the agarose and chicken breast tissue samples; BS, 50/50 beam splitter; Li, ith imaging lens.

First, the reconstructed peak intensity falls as the sample is displaced. This is predictable, as displacements of the sample disrupt the light trajectory retracing condition and cause the reconstruction to deteriorate. Second, the extent to which the reconstructed-peak appeared to be reconstructed is remarkable. For the $0.69-\mathrm{mm}$-thick tissue section, the reconstructed-peak intensity difference between zero sample displacement (optimal reconstruction condition) and large sample displacement of $8 \mu \mathrm{m}$ (mismatched sample and OPC light field) is more than three orders of magnitude. Third, the rate of reconstructed peak intensity drop due to sample lateral translation is a function of the sample thickness. For a 0.69-mm-thick chicken tissue section, a displacement of $\sim 0.7 \mu \mathrm{m}$ results in peak intensity reduction by one order of magnitude. Finally, the reconstruction appears surprisingly robust. With our thickest tissue section of $0.69 \mathrm{~mm}$, the reconstructed-peak intensity was still a respectable $17 \pm 3 \%$ of the agarose reconstructed peak intensity.

The dependence of specific reconstruction efficiency on tissue thickness can be seen from Fig. 4b. We plotted the relative reconstructed-peak intensity on the same scale for zero sample displacement. We can realistically expect the loss of scattering information from light being absorbed by the tissue and/or scattered away from the crystal to cause the reconstruction to deteriorate. Simplistically, we can conjecture that the reconstructed peak height with no sample displacement will follow a Beer's law type of dependency on tissue thickness for thin tissue sections. In other words, the reconstructed peak intensity $I_{\text {peak }}$ is proportional to $\exp (-\alpha L)$, where $\alpha$ is the coefficient associated with the drop in reconstruction efficiency and $L$ is the thickness of the tissue section. Figure $4 \mathrm{~b}$ reveals that such a dependency does indeed hold well for the thin tissue sections $(\sim 0.46 \mathrm{~mm}$ or less), for which an experimental fit for $\alpha$ is $1.45 \pm 0.05 \mathrm{~mm}^{-1}$. For thicker tissues, the drop-off in reconstruction efficiency appears to deviate from this trend. The exact behaviour of the reconstruction efficiency is an important subject that deserves further in-depth study. For comparison, we have added a line in Fig. 4b that shows the unscattered light attenuation as a function of tissue depth; this line shows an expected signal drop associated with coherence-based interference detection methods. In this case, $\mu_{\mathrm{S}}$, the coefficient associated with the drop in reconstruction efficiency, is $38 \mathrm{~mm}^{-1}$. We can see from Fig. $4 \mathrm{~b}$ that the rate of TSOPC efficiency drop occurs at a much slower rate, which clearly indicates that the TSOPC phenomenon is able to make good use of multiply scattered light components.

Figure $4 \mathrm{c}$ shows the change in normalized return transmission collected through the objective as a function of sample lateral displacement. This parameter is different from the reconstructed peak intensity in that it tracks the total amount of light that is returned from the sample (and falls within the collection angle $29^{\circ}$ of the objective), even if the reconstruction is imperfect. The data trace for the $0.69-\mathrm{mm}$-thick section is particularly revealing. Comparing the normalized transmission between when TSOPC is present (zero sample displacement) and absent ( $\sim 2.5 \mu \mathrm{m}$ sample displacement), we can see that TSOPC pushes the total light transmission up by a factor of 3.8. This clearly indicates that TSOPC can be used to achieve enhanced light transmission through biological tissues.

Figure $5 \mathrm{a}$ plots the $1 / \mathrm{e}^{2}$ width of the reconstructed spot as a function of sample displacement. Under optimal reconstruction conditions (zero sample displacement), the reconstructed spot is 

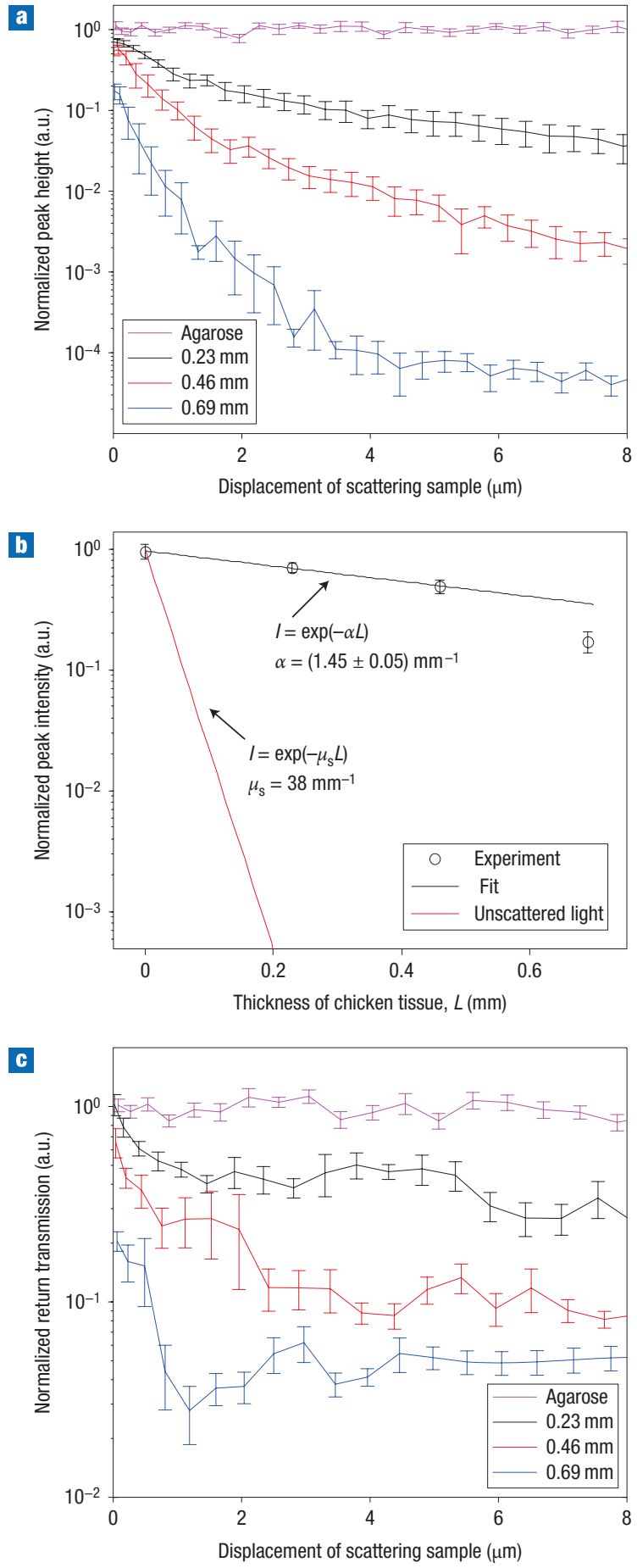

Figure 4 Strength of the reconstructed light field under OPC and non-OPC conditions. a,b, Normalized peak intensity of the reconstructed spot versus the displacement of chicken breast tissue sections of varied thicknesses (a) and versus the thickness of the chicken breast tissue sections for zero displacement (b). In part $\mathbf{b}, I$ is the normalized peak intensity of the reconstructed spot and $\alpha$ is the coefficient associated with the reconstruction efficiency drop. For comparison, the red line shows the expected signal drop associated with coherence-based detection methods. c, Normalized return transmission reaching the CCD, within a collection angle of $29^{\circ}$ from the PCM, versus the displacement of chicken breast tissue sections of different thicknesses. The error bars in each panel represent the standard error of the mean.
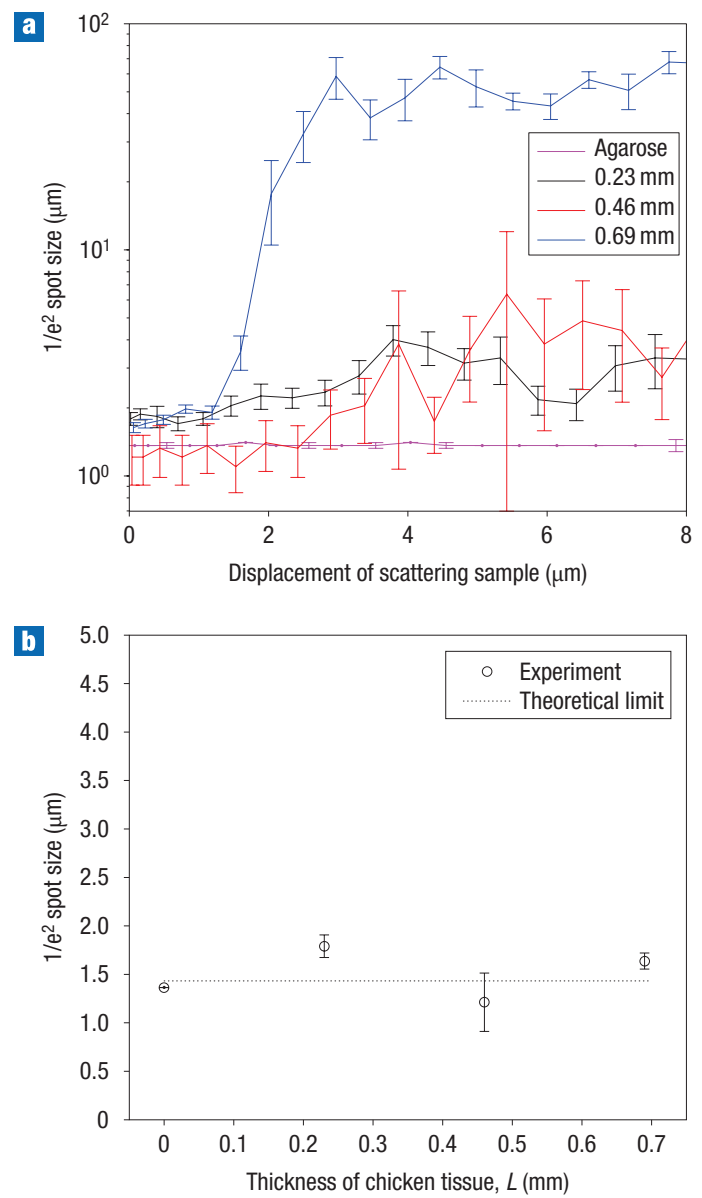

Figure 5 Quality of the reconstructed light field under OPC and non-OPC conditions. $\mathbf{a}, \mathbf{b}, 1 / \mathrm{e}^{2}$ size of the reconstructed spot versus the displacement of chicken tissue sections of different thicknesses (a) and versus the thickness of the chicken tissue sections for zero displacement (b). Part b illustrates that the reconstructed spot size for the ideal case (no sample displacement) is independent of the tissue thickness for tissues of up to $0.69 \mathrm{~mm}$ in thickness. The error bars in each panel represent the standard error of the mean.

tight for all the sample thicknesses, as also shown in Figs $3 \mathrm{~b}$ and $5 \mathrm{~b}$. However, as the displacement increases, the $1 / \mathrm{e}^{2}$ spot size widens. The results in Fig. 5a also indicate that the rate of $1 / \mathrm{e}^{2}$ width increase with sample displacement is a function of sample thickness or scattering extent. Further, we can see from the plots in Fig. 5a, as well as Fig. $3 \mathrm{~b}-\mathrm{f}$, that the reconstructed light spot appeared to consist of two components-a sharp well-reconstructed spot and a more diffused spot. The more diffused spot grew in strength as the sample was displaced and probably consisted of light components that were weakly reconstructed. In comparison, the wellreconstructed spot dropped in intensity with sample displacement but did not appear to increase in width significantly.

More intriguingly, Fig. $5 \mathrm{~b}$ reveals that the $1 / \mathrm{e}^{2}$ width for all tissue thicknesses at zero tissue displacement is approximately the same $(1.5 \mu \mathrm{m})$. This is in good agreement with the estimated $1 / \mathrm{e}^{2}$ spot size of $1.4 \mu \mathrm{m}$ for perfect reconstruction. The insensitivity to tissue thickness and the good fit to the theoretically predicted width for ideal reconstruction suggest that there exists a phase conjugate component that can retrace the initial light trajectory optimally, even if the phase conjugation process as a whole is necessarily imperfect owing to experimental constraints. 


\section{DISCUSSION}

There are numerous ways to generate an OPC light field, including static holography ${ }^{13,14}$, stimulated scattering ${ }^{15,16}$, real-time holography ${ }^{17,18}$, four-wave mixing ${ }^{19,20}$ and six-wave mixing ${ }^{21}$. One can also conceivably combine a wavefront sensor, such as a digital holography system (see Supplementary Information, Fig. S2) or a Shack-Hartman array, with a spatial light modulator to create an electronically controllable PCM. Our experiments used a static holographic approach, as this allowed us to defer the OPC light-field playback-a feature needed for the sample-OPC light-field mismatch experiment.

Dynamic OPC light-field generation methods, such as four-wave mixing $^{19,20}$ or an electronically controllable PCM, are particularly promising as the means for implementing TSOPC-based applications. As there is no appreciable time delay between light-field recording and playback in these methods, the effect of minute movements of cellular components and fluid tissues in living tissue, which can disrupt the reconstruction condition, is effectively nullified. On the other hand, the sensitivity of static holographybased OPC schemes to minute movements can be adapted to enable high-sensitivity movement measurements. In addition, we note that although the crystal chosen for these experiments erases itself with time and light exposure, there exist permanent holographic recording media, such as self-developing photopolymer-based recording media ${ }^{22}$.

The applications of TSOPC are potentially wide ranging. For example, it may be used to enhance light delivery for therapeutic purposes such as photodynamic therapy, in a manner analogous to the use of the ultrasound-based time-reversal phenomenon to deliver high ultrasound dosages for kidney stone ${ }^{10}$ removal. The ability of the TSOPC to enhance light transmission could potentially be used to improve the depth penetration of imaging methods such as photoacoustic tomography ${ }^{23,24}$.

To summarize, we have demonstrated tissue turbidity suppression with OPC. In our experiments, the mean number of scattering events reached up to 26 for the $0.69-\mathrm{mm}$-thick chicken tissue and a significant fraction of the light field never reached the photorefractive crystal $(27 \%$ of the light was backscattered by the 0.69 -mm-thick chicken tissue section). In spite of issues with tissue absorption and incomplete optical-wavefront recording/readout, this newly observed biophotonics phenomenon has proved to be remarkably robust.

Our experiments demonstrate that it is possible to reconstruct a point source caused to deteriorate by passage through $0.69-\mathrm{mm}$-thick tissue such that it is more than three orders of magnitude above the background. In addition, the amount of tolerable tissue and OPC field mismatch is relatively large $(\sim 0.7 \mu \mathrm{m}$ for an order of magnitude reconstruction drop for 0.69 -mm-thick chicken breast tissue section). This is a relevant practical consideration for certain applications. Our experiments also indicate that for zero displacement of the tissue sections, the phenomenon roughly obeys a Beer's law type of dependence on tissue thickness (attenuation coefficient $=1.45 \pm 0.05 \mathrm{~mm}^{-1}$ ) for thin tissue sections. In addition, we found that the quality of the reconstruction (as measured by the width of an optimal reconstructed peak) is independent of tissue thickness for thicknesses up to $0.69 \mathrm{~mm}$; this point is surprising as a deterioration in reconstruction quality would reasonably be expected. Finally, we can enhance overall tissue transmission with TSOPC; we observed an increase in transmission by a factor of 3.8 compared with non-TSOPC transmission for 0.69 -mm-thick tissue within a collection angle of $29^{\circ}$.

\section{METHODS}

The experimental set-up for generating the OPC light field is shown in Fig. 1a. Light from a 532-nm DPSS laser (Coherent) was spatially filtered and collimated
( $1 / \mathrm{e}^{2}$ beam diameter $\left.7.8 \mathrm{~mm}\right)$. This beam then passed through a half-wave plate (WP1) and was split into two by a polarization beam splitter (P1). The reflected (vertically polarized) and the transmitted (horizontally polarized) beams formed the sample arm and reference arms, respectively. Another half-wave plate (WP2), oriented at $45^{\circ}$, changed the polarization state of the reference beam to vertical, causing it to reflect from the second polarization beam splitter (P2) and reach the photorefractive crystal. The combined reference and signal beams then created a holographic pattern in the photorefractive crystal.

To generate the OPC light field, the half-wave plate WP2 was rotated so that the wave-plate direction was coincident with the polarization of the incoming reference beam. The outgoing light remained horizontally polarized, passed straight through the polarization beam splitter $\mathrm{P} 2$, and reached the photorefractive crystal by means of mirrors M2, M3 and M4 to read out the stored hologram. A half-wave plate (WP3) oriented at $45^{\circ}$, placed in the path of the read beam, changed its polarization back to vertical. The position and orientation of each mirror were adjusted so that the read beam was not only coincident with the recording beam but counter-propagating as well. In this geometry, an OPC light field was generated that retraced the path of the original light field and was detected by means of a CCD though lenses L4 and L5 in Fig. 1a. The reconstruction process of the conjugate signal beam using a conjugate reference beam is shown schematically in Fig. 1c.

Received 9 August 2007; accepted 10 December 2007; published 27 January 2008.

References

1. Cheong, W. F., Prahl, S. A. \& Welch, A. J. A review of the optical properties of biological tissues. IEEE J. Quantum Electron. 26, 2166-2185 (1990).

2. Leith, E. N. \& Upatneiks, J. Holographic imagery through diffusing media. J. Opt. Soc. Am. 56, 523 (1966).

3. Levenson, M. D. High-resolution imaging by wave-front conjugation. Opt. Lett. 5, 182-184 (1980).

4. McFarlane, R. A. \& Steel, D. G. Laser-oscillator using resonator with self-pumped phase-conjugate mirror. Opt. Lett. 8, 208-210 (1983).

5. Gower, M. C. KrF laser-amplifier with phase-conjugate Brillouin retro-reflectors. Opt. Lett. 7, $423-425$ (1982).

6. Tomov, I. V., Fedosejevs, R., McKen, D. C. D., Domier, C. \& Offenberger, A. A. Phase conjugation and pulse-compression of KrF-laser radiation by stimulated Raman-scattering. Opt. Lett. 8, 9-11 (1983).

7. Leith, E. et al. Imaging through scattering media with holography. J. Opt. Soc. Am. A 9, $1148-1153$ (1992).

8. Jones, R. et al. Holographic storage and high background imaging using photorefractive multiple quantum wells. Appl. Phys. Lett. 69, 1837-1839 (1996).

9. Cuche, E., Bevilacqua, F. \& Depeursinge, C. Digital holography for quantitative phase-contrast imaging. Opt. Lett. 24, 291-293 (1999).

10. Fink, M. Time-reversed acoustics. Sci. Am. 281, 91-97 (1999).

11. Lerosey, G., De Rosny, J., Tourin, A. \& Fink, M. Focusing beyond the diffraction limit with far-field time reversal. Science 315, 1120-1122 (2007).

12. Mittra, R. \& Habashy, T. M. Theory of wave-front-distortion correction by phase conjugation. J. Opt. Soc. Am. A 1, 1103-1109 (1984).

13. Gabor, D. A new microscopic principle. Nature 161, 777-778 (1948).

14. Lukosz, W. Equivalent-lens theory of holographic imaging. J. Opt. Soc. Am. 58, 1084-1091 (1968).

15. Zel'dovich, B. Y., Popovich, V. I., Ragul'skii, V. V. \& Faizullov, F. S. Connection between wave fronts of reflected and exciting light in stimulated Mandelshtam-Brillouin scattering. JETP Lett. USSR 15, 109-113 (1972).

16. Hellwarth, R. W. Theory of phase conjugation by stimulated scattering in a waveguide. J. Opt. Soc. Am. 68, 1050-1056 (1978).

17. Ivakin, E. V., Petrovich, I. P., Rubanov, A. S. \& Stepanov, B. I. Dynamic holograms in amplifying medium. Kvantovaya Elektronika 2, 1556-1558 (1975).

18. Gunter, P. Holography, coherent-light amplification and optical-phase conjugation with photorefractive materials. Phys. Rep. Rev. Sec. Phys. Lett. 93, 199-299 (1982).

19. Yariv, A. Phase conjugate optics and real-time holography. IEEE J. Quantum Electron. 14, 650-660 (1978).

20. Hellwarth, R. W. Generation of time-reversed wave fronts by nonlinear refraction. J. Opt. Soc. Am. 67, $1-3$ (1977).

21. Charra, F. \& Nunzi, J. M. Nondegenerate multiwave mixing in polydiacetylene-Phase conjugation with frequency-conversion. J. Opt. Soc. Am. B 8, 570-577 (1991).

22. Cheben, P. \& Calvo, M. L. A photopolymerizable glass with diffraction efficiency near $100 \%$ for holographic storage. Appl. Phys. Lett. 78, 1490-1492 (2001).

23. Hoelen, C. G. A., de Mul, F. F. M., Pongers, R. \& Dekker, A. Three-dimensional photoacoustic imaging of blood vessels in tissue. Opt. Lett. 23, 648-650 (1998).

24. Wang, X. et al. Noninvasive laser-induced photoacoustic tomography for structural and functional in vivo imaging of the brain. Nature Biotechnol. 21, 803-806 (2003).

\section{Acknowledgements}

This work was supported by the National Science Foundation career award BES-0547657 and the Defense Advanced Research Projects Agency Center for Optofluidic Integration.

Correspondence and requests for materials should be addressed to C.Y.

Supplementary information accompanies this paper on www.nature.com/naturephotonics.

\section{Author contributions}

Z.Y. was responsible for project planning, experimental work and data analysis. D.P. provided advice and loaned some equipment for the project. M.S.F. provided advice. C.Y. was responsible for administering the project, project planning, data analysis, and obtaining financial support for the project.

Reprints and permission information is available online at http://npg.nature.com/reprintsandpermissions/ 